\title{
Domain-representable spaces
}

\author{
by \\ Harold Bennett (Lubbock, TX) and \\ David Lutzer (Williamsburg, VA)
}

\begin{abstract}
We study domain-representable spaces, i.e., spaces that can be represented as the space of maximal elements of some continuous directed-complete partial order (= domain) with the Scott topology. We show that the Michael and Sorgenfrey lines are of this type, as is any subspace of any space of ordinals. We show that any completely regular space is a closed subset of some domain-representable space, and that if $X$ is domain-representable, then so is any $G_{\delta}$-subspace of $X$. It follows that any Cechcomplete space is domain-representable. These results answer several questions in the literature.
\end{abstract}

1. Introduction. Approximability by special partially ordered sets is an idea that came into topology from theoretical computer science. Contemporary discussions containing many important examples can be found in the papers of Martin, Mislove, and Reed [8], [11], [12]. An article by Ciesielski, Flagg, and Kopperman [4] gives valuable motivation for studies of this type (and proves important results about countably-based models of spaces).

Because the literature about domain-representations contains conflicting terminology, we begin with the basic definitions to be used in this paper. Let $(P, \sqsubseteq)$ be a partially ordered set (= poset). A subset $D \subseteq P$ is directed provided for each $d_{1}, d_{2} \in D$, some $d_{3} \in D$ has $d_{1}, d_{2} \sqsubseteq d_{3}$. We say that such a $d_{3}$ is a common extension of $d_{1}$ and $d_{2}$. For any set $T \subseteq P$ we write $\sup (T)$ to mean the least of all of the upper bounds of $T$ in $P$, if such a thing exists. Most frequently $T$ will be a directed set, but sometimes $T$ will be a subset of $P$ with the property that if $t_{1}, t_{2} \in T$ then some $p \in P$ has $t_{1}, t_{2} \sqsubseteq p$. The poset $(P, \sqsubseteq)$ is a dcpo (= directed-complete partial order) if every nonempty directed set $D \subseteq P$ has a supremum in $P$. If $(P, \sqsubseteq)$ is a

2000 Mathematics Subject Classification: Primary 54E52; Secondary 06B35, 06F30, $54 \mathrm{~F} 05$.

Key words and phrases: continuous dcpo, domain, Scott domain, domain-representable space, Baire space, Choquet-complete space, Michael line, Sorgenfrey line, ordinal space, $G_{\delta}$-subspace, Čech-complete space. 
dcpo, then Zorn's Lemma shows that for each $p \in P$ at least one maximal element $x \in P$ has $p \sqsubseteq x$. The set of all maximal elements of $P$ is denoted by $\max (P)$.

Starting with a poset $(P, \sqsubseteq)$, there is a new relation $\ll$ on $P$ defined as follows: for $p, q \in P, p \ll q$ means that for any directed set $D$ with $q \sqsubseteq \sup (D)$, there is some $d \in D$ with $p \sqsubseteq d$. Traditionally $p \ll q$ is read " $p$ is far below $q$ " or " $q$ is far above $p$ ". Clearly $p \ll q$ implies $p \sqsubseteq q$ (use the directed set $D=\{q\}$ in the definition of $p \ll q)$. It can happen that some $p \in P$ has $p \ll p$ and such elements of $P$ are called compact elements of $P$. For $p \in P$ we write

$$
\Uparrow(p)=\{q \in P: p \ll q\} \quad \text { and } \quad \Downarrow(p)=\{q \in P: q \ll p\} .
$$

We say that the poset $(P, \sqsubseteq)$ is continuous if for each $p \in P$, the set $\Downarrow(p)$ is directed and has $p=\sup (\Downarrow(p))$. The word domain used in the title is a synonym for "continuous dcpo". To say that a domain is algebraic means that for each $p \in P$, the set $K(p)$ of compact elements of $P$ that belong to $\Downarrow(p)$ is a directed set with $\sup (K(p))=p$.

We introduce a topology on $P$ as follows. A subset $U$ of a dcpo $(P, \sqsubseteq)$ is Scott-open provided $U$ has the following two properties:

(a) if $x \in U$ and $x \sqsubseteq y \in P$, then $y \in U$;

(b) if $D \subseteq P$ is any directed set with $\sup (D) \in U$ then $D \cap U \neq \emptyset$.

The collection of all Scott-open sets is a topology on the set $P$, called the Scott topology. From a traditional topological viewpoint, the Scott topology is not a good one - as shown in Proposition 2.6 of [12], it is always $T_{0}$ but almost never $T_{1}$. However, when restricted to the subset $\max (P)$, the Scott topology can be very nice, as can be seen from Theorem 1.1 below. If a topological space $X$ is homeomorphic to the space $\max (P)$ topologized using the relative Scott topology for some continuous dcpo $(P, \sqsubseteq)$, then we say that the space $X$ is representable as the space of maximal elements of $P$ and that $X$ is domain-representable.

Being representable as the space of maximal elements of some domain is a nontrivial restriction on a space $X$. For example, any such space $X$ is a Baire space, and (even more) is Choquet-complete [10]. (Choquet completeness is a game-theoretic property that is equivalent to Cech-completeness in any metric space; see Theorem 8.7 in [3] and Choquet's remarks after the proof of (8.7) there.)

Many important types of spaces are representable as the space of maximal elements of some continuous dcpo. The following theorem summarizes results of Edalat and Heckmann [5] and Martin [10] concerning complete metrizability, and of Gierz et al. [7] concerning local compactness. See also Examples 3.6 and 3.7 in [8] and Example 3.7 in [11]. 
Theorem 1.1. A metrizable space $X$ is representable as the space of maximal elements of some continuous dcpo if and only if $X$ is completely metrizable, and any locally compact Hausdorff space is representable as the space of maximal elements of some continuous dcpo.

The goal of our paper is to study spaces that can be represented as the space of maximal elements in a continuous dcpo and to extend Theorem 1.1 in a significant way by answering several questions in the literature. We will show:

(1) Representability as the space of maximal elements of some domain is not a closed-hereditary property. In fact, any completely regular $T_{1}$ space $Y$ is a closed subspace of a completely regular $T_{1}$-space $X(Y)$ that is domain-representable. This answers a question posed in [9].

(2) Suppose $X=\max (P)$ for some continuous dcpo $P$. If $Y$ is a $G_{\delta^{-}}$ subset of $X$, then $Y$ is also domain-representable. In particular, every Cech-complete space is domain-representable. (This answers question (vii) in [8].) Furthermore, if $X$ is both perfect (= closed sets are $G_{\delta^{-}}$-sets) and domain-representable, then so is any closed subset of $X$.

(3) Both the Michael line and the Sorgenfrey line can be represented as the space of maximal elements of some continuous dcpo, and so can any subspace of any ordinal.

We will use several known results about dcpos at many points in this paper, so we record them here. The first is easy and the other two appear in the literature.

Lemma 1.2. If $(D, \sqsubseteq)$ is any nonempty directed set that contains no maximum element, then for each $d \in D$ there exists a sequence $\left\langle d_{j}\right\rangle$ of distinct elements of $D$ with $d=d_{1} \sqsubseteq d_{2} \sqsubseteq \cdots$.

Lemma 1.3 (see [13] or Proposition 2.13 in [8]). Let $(P, \sqsubseteq)$ be a continuous dcpo. Then the collection $\{\Uparrow(p): p \in P\}$ is a basis for the Scott topology on $P$.

Lemma 1.4 (see [13] or Proposition 2.9 in [8]). Suppose $(P, \sqsubseteq)$ is a continuous dcpo and that $a, c \in P$ have $a \ll c$. Then there is some $b \in P$ (possibly $b \in\{a, c\}$ ) with $a \ll b \ll c$.

Throughout this paper, we will reserve the symbols $\mathbb{R}, \mathbb{Q}$ and $\mathbb{P}$ for the usual sets of real, rational, and irrational numbers, and $\mathbb{Z}$ will denote the set of all integers (positive and negative). In addition, for any ordered pair $p, \pi_{i}(p)$ will denote the $i$ th coordinate of $p$.

The authors would like to thank K. Martin and G. M. Reed for helpful correspondence during the preparation of this paper. We also thank K. P. Hart for comments that substantially improved an earlier draft. 
2. Examples of domain-representable spaces. This section contains a technical construction that will be used in later sections, as well as some examples that may be of interest in themselves.

Proposition 2.1. Suppose that the topological space $(X, \sigma)$ has $X=$ $\max (P)$ where $(P, \sqsubseteq)$ is a continuous dcpo, and suppose that $I$ is an arbitrary subset of $X$. Use $\sigma \cup\{\{y\}: y \in I\}$ as the base for a new topology $\tau$ on $X$. Then there is a continuous dcpo $(Q, \preceq)$ having $\max (Q)=(X, \tau)$.

Proof. For each $y \in I$, let $y^{+}$be any point not in $P$, chosen in such a way that if $y \neq z$ are in $I$, then $y^{+} \neq z^{+}$. Let $Q=P \cup\left\{y^{+}: y \in I\right\}$. Thinking of partial orders as sets of ordered pairs, we define an ordering $\preceq$ on $Q$ by

$$
\preceq=\sqsubseteq \cup\left\{\left(p, y^{+}\right): p \sqsubseteq y\right\} \cup\left\{\left(y, y^{+}\right): y \in I\right\} .
$$

Then $\preceq$ is a partial ordering of $Q$ and it is clear that, as a set, $\max (Q)=$ $\{y: y \in X-I\} \cup\left\{y^{+}: y \in I\right\}$. We will identify this set with $X$ in the obvious way.

We claim that $(Q, \preceq)$ is a dcpo. We must show that if $D$ is a nonempty directed subset of $Q$, then $D$ has a supremum in $Q$. In case $D \subseteq P$, some $p_{0} \in P$ is the supremum of $D$ in $(P, \sqsubseteq)$ and therefore also in $(Q, \preceq)$. In case $D$ is not a subset of $P$, there is some $y^{+} \in Q$ with $y^{+} \in D$, and directedness of $D$ guarantees that this $y^{+}$is unique. But then $y^{+}$is the supremum of $D$ in $(Q, \preceq)$.

Next we claim that $Q$ is continuous. In this part of the proof, $\Downarrow_{\sqsubseteq}(r)$ (respectively, $\Downarrow_{\preceq}(r)$ ) will denote the collection of members of $P$ (respectively, of $Q$ ) that are far below $r$ in the poset $(P, \sqsubseteq)$ (respectively, in $(Q, \preceq)$ ). We must show that $r=\sup \left(\Downarrow_{\prec}(r)\right)$ for each $r \in Q$, where the supremum is taken in $Q$, and that $\Downarrow_{\preceq}(r)$ is directed. In case $r \in P$, both are automatic. To complete this part of the proof, we will show that $y^{+} \in \Downarrow_{\prec}\left(y^{+}\right)$for each $y \in I$. To that end, suppose $E$ is a directed subset of $Q$ and that $y^{+} \preceq \sup _{\preceq}(E)$ where the supremum is taken in $(Q, \preceq)$. If $y^{+} \in E$ there is nothing to prove, so assume $y^{+} \notin E$. For any $e \in E$ we know that $e \preceq y^{+}$so that the definition of $\preceq$ gives $y \in P$ and $e \sqsubseteq y$. But then $y$ is an upper bound for $E$ in $(Q, \preceq)$ that is strictly below $y^{+}$, and that is impossible. Therefore $y^{+} \in \Downarrow_{\preceq}\left(y^{+}\right)$for each $y^{+} \in Q$. Hence $y^{+}$is a common extension of any two members of $\Downarrow_{\preceq}\left(y^{+}\right)$, so that $\Downarrow_{\preceq}\left(y^{+}\right)$is directed and $y^{+}=\sup _{\preceq}\left(\Downarrow\left(y^{+}\right)\right)$, as required.

As noted above, $\max (Q)=\{y: y \in X-I\} \cup\left\{y^{+}: y \in I\right\}$. To complete the proof, we show that the relative Scott topology on $\max (Q)$ is the topology $\tau$ described in the statement of the proposition. Suppose $z \in U \in \tau$. If $z \in I$, then $z$ is identified with the point $z^{+} \in \max (Q)$ and because we know that $z^{+} \in \Downarrow_{\preceq}\left(z^{+}\right)$we also have $z^{+} \in \Uparrow \preceq\left(z^{+}\right)$. Hence $\Uparrow_{\preceq}\left(z^{+}\right) \cap \max (Q)=\left\{z^{+}\right\}$, showing that $U$ is a neighborhood of $z$ in the rel- 
ative Scott topology. Next suppose $z \in U \in \tau$ with $z \in X-I$. Then $U$ is a neighborhood of $z$ in the original topology $\sigma$ so that there is some $p \in P$ with $z \in \Uparrow \sqsubseteq(p)$ and $\Uparrow_{\sqsubseteq}(p) \cap \max (P) \subseteq U$. But then $z \in \Uparrow_{\preceq}(p) \cap \max (Q) \subseteq U$, as required to show that $U$ is relatively Scott-open. Conversely, consider any set $\Uparrow_{\prec}(r) \cap \max (Q)$ where $r \in Q$. If $r \in P$, this is the same as $\Uparrow_{\sqsubset}(r) \cap \max (P)$, which is open in $\sigma$ and hence also in $\tau$. In case $r \notin P$, we have $r=y^{+}$for some $y \in I$ and so $\Uparrow_{\prec}(r) \cap \max (Q)=\left\{y^{+}\right\} \in \tau$. Thus, every basic open set in the relative Scott topology on $\max (Q)$ is $\tau$-open. Hence the relative Scott topology on $\max (Q)$ coincides with $\tau$, as required.

Generalized ordered spaces (GO-spaces) are Hausdorff spaces $(X, \tau)$ that admit a linear order $<$ such that there is a base for $\tau$ consisting of orderconvex sets. An easy way to obtain GO-spaces is to start with some linearly ordered set $(X,<)$ and choose three disjoint subsets $R, L$, and $I$ of $X$. Isolate each point of $I$, and let basic neighborhoods of any point $x \in R$ have the form $[x, b)$ for $x<b \in X$. Basic neighborhoods of points of $y \in L$ have the form $(a, y]$ for $a<y$, and points of $X-(R \cup L \cup I)$ have their usual open interval neighborhoods. GO-spaces have been widely used as sources of examples in topology. The most famous are the Michael and the Sorgenfrey lines, as well as spaces (and subspaces) of ordinals.

EXAMPLE 2.2. The Michael line is representable as the space of maximal elements of a continuous dcpo.

Proof. The usual real line is representable as the set of maximal elements of the poset $\{[a, b]: a \leq b, a, b \in \mathbb{R}\}$ ordered by reverse inclusion. Let $I$ be the set of all irrational numbers and apply Proposition 2.1.

It is harder to prove that the Sorgenfrey line is representable as the space of maximal elements of some continuous dcpo, but our next example shows that it is true.

EXAmple 2.3. The Sorgenfrey line $S$ is representable as the space of maximal elements of some continuous dcpo.

Outline of proof. For each pair of real numbers $a<b$, let $S(a, b)$ be a strictly increasing sequence $\left\langle x_{n}\right\rangle$ with $a=x_{1}, \lim _{n \rightarrow \infty} x_{n}=b$, and $\left|x_{n+1}-x_{n}\right| \leq(b-a) / 2$. Let $C_{0}=\mathbb{Z}$ and, given $C_{n}$, let

$$
C_{n+1}=\bigcup\left\{S(a, b): a<b, a, b \in C_{n},(a, b) \cap C_{n}=\emptyset\right\} .
$$

Let $C=\bigcup\left\{C_{n}: 0 \leq n<\omega\right\}$. Note that if $a<b$ are consecutive points of some $C_{n}$, then $b-a \leq 2^{-n}$.

Let $A=\mathbb{R} \times\{0,1\}$ carry the open interval topology of the lexicographic order. For real numbers $a<b$ let $J(a, b)=[(a, 1),(b, 0)]$. Each $J(a, b)$ is a 
compact, convex subset of $A$. Now define

$$
Q(\omega)=\{(\{(x, 0),(x, 1)\}, \omega): x \in \mathbb{R}-C\} \cup\{(\{(x, 1)\}, \omega): x \in \mathbb{R}\}
$$

and for each $n$ with $0 \leq n<\omega$ let

$$
Q(n)=\{(J(a, b), n): a<b, b \in C(n) \text { and }(a, b) \cap C(n)=\emptyset\} .
$$

Let $Q=\bigcup\{Q(n): 0 \leq n \leq \omega\}$. Define a relation $\sqsubseteq$ in $Q$ by the rule that for $q_{1}, q_{2} \in Q, q_{1} \sqsubseteq q_{2}$ if and only if one of the following holds:

(a) $q_{1}=q_{2}$;

(b) for $i=1,2, q_{i}=\left(J\left(a_{i}, b_{i}\right), n_{i}\right) \in Q-Q(\omega), J\left(a_{2}, b_{2}\right) \subseteq J\left(a_{1}, b_{1}\right)$, and $n_{1}<n_{2}$

(c) $q_{1}=(J(a, b), n) \in Q-Q(\omega), q_{2} \in Q(\omega)$ and $q_{2} \subseteq \operatorname{Int}_{A}(J(a, b))$;

(d) $q_{i}=\left(p_{i}, \omega\right)$ and $p_{2} \subseteq p_{1}$.

Then $(Q, \sqsubseteq)$ is a poset. If $E$ is a nonempty directed subset of $Q$ that does not contain its own maximum element, then $E \cap Q(\omega)=\emptyset$ and $\left\{\pi_{1}(e): e \in E\right\}$ is a directed collection of compact, convex subsets of $A$. Lemma 1.2 gives a sequence $e_{1} \sqsubseteq e_{2} \sqsubseteq \cdots$ of distinct elements of $E$ where $\pi_{2}\left(e_{i}\right)<\pi_{2}\left(e_{i+1}\right)$ for each $i$. The width restriction on consecutive points in $C_{n_{i}}$, where $n_{i}=$ $\pi_{2}\left(e_{i}\right)$, forces $\bigcap\left\{\pi_{1}(e): e \in E\right\} \subseteq\{(x, 0),(x, 1)\}$ for some $x \in \mathbb{R}$. If some $e \in E$ has the form $e=(J(x, b), n)$, then $\sup (E)=\{(x, 1)\}$, and otherwise $\sup (E)=\{(x, 0),(x, 1)\}$. Therefore, $(Q, \sqsubseteq)$ is a dcpo.

For any $q \in Q$ with $\pi_{2}(q)<\omega$ we have $q \ll q$ so that $q \in \Downarrow(q)$. Therefore $q$ is a common extension of any two members of $\Downarrow(q)$, making $\Downarrow(q)$ a directed set, and $q=\sup (\Downarrow(q))$. For distinct $q_{1}, q_{2} \in Q(\omega)$ it never happens that $q_{1} \ll q_{2}$, and for any $q \in Q(\omega), \Downarrow(q)=\left\{q^{\prime} \in Q-Q(\omega): q^{\prime} \sqsubseteq q\right\}$. Hence $\Downarrow(q)$ is directed and has $\sup (\Downarrow(q))=q$. Therefore, $Q$ is a continuous dcpo.

The set of maximal elements of $Q$ is given by $\max (Q)=\{\{(x, 1)\}: x \in \mathbb{R}\}$. For each $x \in \mathbb{R}$ there are members $q=(J(x, b), n) \in Q$ with $\epsilon=b-x$ arbitrarily small, so that $\max (Q) \cap \Uparrow(q)=[x, x+\epsilon)$. Hence the topology induced on $\max (Q)$ by the Scott topology on $Q$ is exactly the Sorgenfrey line topology.

ExAMPLE 2.4. Suppose $X$ is any subspace of any space of ordinals. Then $X$ is representable as the space of maximal elements of some continuous dcpo.

Proof. There is a strictly increasing function $f$ from the ordered set $X$ onto some initial ordinal $[0, \beta)$. The function $f$ might fail to be a homeomorphism from $X$ with its given topology onto $[0, \beta)$ with its usual open interval topology because for some isolated point $x \in X, f(x)$ might be a limit ordinal in $[0, \beta)$. Let $I=\{f(x): x \in X$ is isolated and $f(x)$ is a limit ordinal $\}$. Because, in its usual order topology, $[0, \beta)$ is locally compact, it is representable as $\max (P)$ for come continuous dcpo $(P, \sqsubseteq)$. Let $Y$ be the space 
obtained by isolating every point of the set $I$. In the light of Proposition 2.1, the space $Y$ is representable as the subspace of maximal elements of some continuous dcpo, and $Y$ is homeomorphic to $X$.

Subspaces of ordinals are not the only spaces that are hereditarily domain-representable. In a subsequent paper, we will show that if $X$ is a countable $T_{1}$-space with exactly one non-isolated point, then every subspace of $X$ is homeomorphic to the space of maximal elements of some continuous dcpo with the Scott topology.

QUESTION 2.5. For which spaces $X$ is it true that every subspace of $X$ is representable as the space of maximal elements of some continuous dcpo with the Scott topology? Must such an $X$ be scattered?

3. Subspaces of domain-representable spaces. One can think of domain-representability as a kind of topological completeness property, related to being a Baire space. The property of being a Baire space, and most other completeness properties associated with being a Baire space, are open-hereditary properties and are hereditary to dense $G_{\delta}$-sets, but are not closed-hereditary. (See [1].) In addition, if $X$ has a dense subspace $Y$ that is a Baire space, then $X$ is also a Baire space. In this section we show that representability as the space of maximal elements of some continuous dcpo follows that same pattern to a large degree, but not entirely.

EXAMPLe 3.1. Any completely regular space $Y$ is a closed subset of a space $X$ that can be represented as the space of maximal elements of a continuous dcpo. Hence there is a space $X$ that is representable as the space of maximal elements of a continuous dcpo and a closed subset $Y \subseteq X$ that cannot be represented as the space of maximal elements of any continuous dcpo.

Proof. Given a completely regular space $Y$, we know that the compact Hausdorff space $\beta Y$ can be represented as the space of maximal elements of some continuous dcpo (see Theorem 1.1). Let $X$ be the space that is obtained from $\beta Y$ by isolating all points of $\beta Y-Y$. By Proposition 2.1, $X$ is also representable as the space of maximal elements of some continuous dcpo, and $Y$ is a closed subspace of $X$. To obtain the second assertion of the example from the first, let $\mathbb{Q}$ be the usual set of rational numbers, construct $\beta \mathbb{Q}$ and isolate all points of $\beta \mathbb{Q}-\mathbb{Q}$. Because $\mathbb{Q}$ is not a Baire space, it cannot be represented as the space of maximal elements of a continuous dcpo. (The second assertion also follows from Example 2.2.)

Example 3.1 answers a question of K. Martin [9]. While domain-representability is not a closed-hereditary property, it is open-hereditary. (According to an e-mail from K. Martin, Reinhold Heckmann was the first to 
observe that domain-representability is an open-hereditary property.) Our next result goes much further.

Theorem 3.2. Suppose that a space $X$ can be represented as the space of maximal elements of some continuous dcpo $(P, \sqsubseteq)$ and that $Y$ is a $G_{\delta^{-}}$ subspace of $X$. Then there is a continuous dcpo $(Q, \preceq)$ such that $Y$ is homeomorphic to the space $\max (Q)$. Furthermore, $(Q, \preceq)$ is algebraic.

Proof. Write $X=\max (P, \sqsubseteq)$. Because $Y$ is a $G_{\delta}$-subset of $X$, there must be Scott-open subsets $O(n)$ of $P$ with $O(n+1) \subseteq O(n)$ and $Y=$ $X \cap \bigcap\{O(n): n<\omega\}$. Let

$$
Q_{n}=\{(p, n): p \in O(n) \text { and } \Uparrow(p) \cap Y \neq \emptyset\}
$$

and define

$$
Q_{\omega}=\{(p, \omega): p \in \bigcap\{O(n): n<\omega\}\} .
$$

Let $Q=\bigcup\left\{Q_{n}: n \leq \omega\right\}$. Notice that if $y \in Y$, then $(y, \omega) \in Q$.

We claim that $(p, \omega) \in Q_{\omega}$ if and only if for each $n<\omega$ we have $\Downarrow(p) \cap O(n) \neq \emptyset$. For suppose that $(p, \omega) \in Q_{\omega}$. Then for each $n<\omega$ we have $p \in O(n)$ so that for some $p_{n} \in P$ we have $p \in \Uparrow\left(p_{n}\right) \subseteq O(n)$. The Interpolation Lemma 1.4 gives us some $p_{n}^{\prime} \in P$ with $p_{n} \ll p_{n}^{\prime} \ll p$ so that $p_{n}^{\prime} \in \Downarrow(p) \cap O(n)$, showing that $\Downarrow(p) \cap O(n) \neq \emptyset$ for each $n<\omega$. Conversely, if $\Downarrow(p) \cap O(n) \neq \emptyset$ for each $n<\omega$, then some $p_{n}$ has $p_{n} \ll p$ and $p_{n} \in O(n)$ so that, $O(n)$ being Scott-open, we have $p \in O(n)$.

We define a relation $\preceq$ on $Q$ by the rule that if $(p, m),(q, n) \in Q$ (with $m, n \leq \omega)$, then $(p, m) \preceq(q, n)$ if and only if one of the following holds:

(a) $(p, m)=(q, n)$;

(b) $m<n<\omega$ and $p \ll q$;

(c) $p \ll q$ and $m<n=\omega$;

(d) $p \sqsubseteq q$ and $m=n=\omega$.

Then $\preceq$ is a partial order on $Q$ and the relation $\left(p_{1}, \omega\right) \preceq\left(p_{2}, n_{2}\right)$ never happens for $n_{2}<\omega$.

The rest of the proof involves a sequence of steps. We will carefully distinguish between the partial orders $\sqsubseteq$ and $\preceq$, but we will use $\ll$ to denote the "far below" relation in both $(P, \sqsubseteq)$ and $(Q, \preceq)$, relying on context to make the meaning clear. In addition, we will write $\sup (S)$ meaning the supremum of $S$ in $P$ or $Q$, depending upon whether $S \subseteq P$ or $S \subseteq Q$. Similarly we will write $\Downarrow(x)$ rather than $\Downarrow_{P}(x)$ or $\Downarrow_{Q}(x)$ and are confident that readers will know which we mean by deciding whether $x \in P$ or $x \in Q$.

STEP 1: $(Q, \preceq)$ is a dcpo. We will show that if $E$ is a nonempty directed subset of $Q$, then $\sup (E) \in Q$. If $E$ contains a maximal element $e^{*}$ of itself then $\sup (E)=e^{*} \in Q$ so suppose that no point of $E$ is maximal in $E$. The 
set $\pi_{1}[E]=\left\{\pi_{1}(e): e \in E\right\}$ is a nonempty directed subset of $P$, so that some $p^{*} \in P$ has $p^{*}=\sup \left(\pi_{1}[E]\right)$.

We claim that $\left(p^{*}, \omega\right) \in Q$. There are two cases to consider. In the first, suppose $E \cap Q_{\omega} \neq \emptyset$. Then some $e \in E$ has $e=(p, \omega)$ so that $e \in Q$ yields $p \in \bigcap\{O(n): n<\omega\}$. Then $p \sqsubseteq p^{*}$ yields $p^{*} \in \bigcap\{O(n): n<\omega\}$ because each $O(n)$ is Scott-open, and therefore $\left(p^{*}, \omega\right) \in Q$. In the second case, suppose that $E \cap Q_{\omega}=\emptyset$. Choose distinct $e_{i}=\left(p_{i}, n_{i}\right) \in E$ with $e_{i} \preceq e_{i+1}$. Then $n_{i}<n_{i+1}$ so that $p_{i} \in O\left(n_{i}\right)$. Since $p_{i} \sqsubseteq p^{*}$, we have $p^{*} \in O\left(n_{i}\right)$ because each $O\left(n_{i}\right)$ is Scott-open. Hence

$$
p^{*} \in \bigcap\left\{O\left(n_{i}\right): i \geq 1\right\}=\bigcap\{O(n): n \geq 1\},
$$

showing that $\left(p^{*}, \omega\right) \in Q$.

Knowing that $q^{*}=\left(p^{*}, \omega\right) \in Q$, we can show that $q^{*}$ is an upper bound for $E$ in $Q$. For let $e \in E$. In case $e=(p, n)$ with $n<\omega$, some $e_{1} \in E-\{e\}$ has $e \preceq e_{1}$. According to part (b) or (c) of the definition of $\preceq$, we must have $\pi_{1}(e) \ll \pi_{1}\left(e_{1}\right) \sqsubseteq \sup \left(\pi_{1}[E]\right)=p^{*}$ so that $\pi_{1}(e) \ll p^{*}$ and therefore $e \preceq\left(p^{*}, \omega\right)=q^{*}$. In case $e=(p, \omega)$, we have $\pi_{1}(e) \sqsubseteq \sup \left(\pi_{1}[E]\right)=p^{*}$ and part (d) of the definition of $\preceq$ shows that $e \preceq\left(p^{*}, \omega\right)=q^{*}$.

We complete the proof that $\sup (E)=q^{*}$ by showing that $q^{*} \preceq q$ whenever $q$ is an upper bound for $E$ in $Q$. Given an upper bound $q$ we know that $\pi_{1}(e) \sqsubseteq \pi_{1}(q)$ for each $e \in E$. Because $p^{*}=\sup \left(\pi_{1}[E]\right)$ it follows that $p^{*}=\pi_{1}\left(q^{*}\right) \sqsubseteq \pi_{1}(q)$. Either because some $e \in E$ has $\pi_{2}(e)=\omega$, or because we can find distinct $e_{i} \in E$ with $\pi_{2}\left(e_{i}\right)<\pi_{2}\left(e_{i+1}\right)$, we know that $\pi_{2}(q)=\omega$. But then $\pi_{1}\left(q^{*}\right) \sqsubseteq \pi_{1}(q)$ is enough to show that $q^{*} \preceq q$, as required.

SteP 2: If $(p, n) \in Q$ with $n<\omega$, then $(p, n) \ll(p, n)$ in $Q$. To verify that assertion, suppose $E$ is a nonempty directed set in $Q$ with $(p, n) \preceq$ $\sup (E)$. The nontrivial case is where $E$ contains no maximal element of itself. As proved in Step 1, we then know that $\sup (E)=\left(p^{*}, \omega\right)$ where $p^{*}=\sup \left(\pi_{1}(E)\right)$ in $P$. Then $(p, n) \preceq \sup (E)=\left(p^{*}, \omega\right)$ so that $p \ll p^{*}$ in $P$. Now we invoke Lemma 1.4 to find some $r \in P$ with $p \ll r \ll p^{*}$ in $P$. Because $r \ll p^{*}=\sup \left(\pi_{1}(E)\right)$, some $e_{1}=\left(p_{1}, m_{1}\right) \in E$ has $p \ll r \sqsubseteq \pi_{1}\left(e_{1}\right)$. Either because some element of $E$ has second coordinate $\omega$ or because we can choose distinct points $e_{2}, e_{3}, \ldots$ of $E$ with $e_{1} \preceq e_{2} \preceq e_{3} \preceq \cdots$ and with $\pi_{2}\left(e_{i}\right)<\pi_{2}\left(e_{i+1}\right)<\omega$ for each $i$, we can find $e^{*} \in E$ with $e_{1} \sqsubseteq e^{*}$ and $n<\pi_{2}\left(e^{*}\right)$. But then $(p, n) \preceq e^{*} \in E$ as required to show that $(p, n) \ll(p, n)$.

STEP 3: If $\left(p^{*}, \omega\right) \in Q$ then $\left(p^{*}, \omega\right)=\sup \left(E^{*}\right)$ where $E^{*}=\{(p, n) \in Q$ : $1 \leq n<\omega$ and $\left.p \in \Downarrow\left(p^{*}\right) \cap O(n)\right\}$. To verify that assertion, first note that $\left(p^{*}, \omega\right)$ is clearly an upper bound for $E^{*}$. Next observe that $E^{*} \cap Q(n) \neq \emptyset$ for each fixed $n<\omega$, because $\left(p^{*}, \omega\right) \in Q$ yields $p^{*} \in O(n)$ and then some $r \in P$ with $p^{*} \in \Uparrow(r) \subseteq O(n)$. Lemma 1.4 gives some $s$ with $r \ll s \ll p^{*}$ and then $(s, n) \in E^{*}$. Next consider any upper bound $q \in Q$ for $E^{*}$. We will 
show that $\left(p^{*}, \omega\right) \preceq q$. Because $E^{*} \cap O(n) \neq \emptyset$ for each $n<\omega$, we know that $\pi_{2}(q)=\omega$ so that $q$ has the form $q=(p, \omega)$ for some $p \in P$. Therefore we will have the desired $\left(p^{*}, \omega\right) \preceq q$ provided we can prove $p^{*} \sqsubseteq \pi_{1}(q)$. To do that, we will show that $\pi_{1}(q)$ is an upper bound for $\Downarrow\left(p^{*}\right)$ in $P$, and that will yield $p^{*}=\sup \left(\Downarrow\left(p^{*}\right)\right) \sqsubseteq \pi_{1}(q)$ as required.

Consider any $t \in \Downarrow\left(p^{*}\right)$. We know that $p^{*} \in \bigcap\{O(n): n<\omega\} \subseteq O(1)$, so that there is some $s \in P$ with $p^{*} \in \Uparrow(s) \subseteq O(1)$. Then $s \ll p^{*}$ so that Lemma 1.4 provides some $r \in P$ with $s \ll r \ll p^{*}$. Because the directed set $\Downarrow\left(p^{*}\right)$ contains both $t$ and $r$, some $u \in \Downarrow\left(p^{*}\right)$ has $r, t \sqsubseteq u$. Then $s \ll r \sqsubseteq u$ and so $u \in \Uparrow(s) \subseteq O(1)$. Therefore $u \in O(1) \cap \Downarrow\left(p^{*}\right)$, showing that $(u, 1) \in E^{*}$. Because $q$ is an upper bound for $E^{*}$ we know that $(u, 1) \preceq q$ and therefore $u \ll \pi_{1}(q)$. But then $t \sqsubseteq u \ll \pi_{1}(q)$ gives $t \ll \pi_{1}(q)$ so that $\pi_{1}(q)$ is an upper bound for $\Downarrow\left(p^{*}\right)$. Hence $p^{*} \sqsubseteq \pi_{1}(q)$ as required to show that $\left(p^{*}, \omega\right) \preceq q$. This completes Step 3.

SteP 4: If $(\widehat{p}, \omega),\left(p^{*}, \omega\right) \in Q$, then $(\widehat{p}, \omega) \ll\left(p^{*}, \omega\right)$ never happens in $Q$. Consider the set $E^{*}$ defined using $p^{*}$ in Step 3. We know that $\left(p^{*}, \omega\right)=$ $\sup \left(E^{*}\right)$ so that if $(\widehat{p}, \omega) \ll\left(p^{*}, \omega\right)$, then some $(p, n) \in E^{*}$ would have $(\widehat{p}, \omega) \preceq(p, n)$ and because $n<\omega$ that can never happen.

STEP 5: $(Q, \preceq)$ is a continuous poset, i.e., for each $q \in Q, \Downarrow(q)$ is directed and $q=\sup (\Downarrow(q))$. In case $q$ has the form $q=(p, n)$ with $n<\omega$, Step 2 gives $q \in \Downarrow(q)$ so that $q$ is a common extension of any two members of $\Downarrow(q)$ and $q=\sup (\Downarrow(q))$. Now consider the case where $q=\left(p^{*}, \omega\right) \in Q$. Let $q_{1}, q_{2} \in \Downarrow\left(p^{*}, \omega\right)$. From Step 4 , each $q_{i}$ has the form $q_{i}=\left(p_{i}, n_{i}\right)$ with $n_{i}<\omega$. Because $q_{i} \ll\left(p^{*}, \omega\right)$ in $Q$ and $n_{i}<\omega$, we know that $\pi_{1}\left(q_{i}\right) \ll p^{*}$ in $P$. Let $m=n_{1}+n_{2}$. Because $\left(p^{*}, \omega\right) \in Q$ we may choose $r \in \Downarrow\left(p^{*}\right) \cap O(m)$. Because $p_{1}, p_{2}, r$ belong to the directed set $\Downarrow\left(p^{*}\right)$, some $s \in \Downarrow\left(p^{*}\right)$ has $p_{1}, p_{2}, r \sqsubseteq s$. Because $s \ll p^{*}$ in $P$, Lemma 1.4 gives $t \in P$ with $s \ll t \ll p^{*}$. Because $t \ll p^{*}$ we have $(t, m) \preceq\left(p^{*}, \omega\right)$. Applying Step 3 to $(t, m)$, we obtain $(t, m) \ll(t, m) \preceq\left(p^{*}, \omega\right)$ in $Q$ so that $(t, m) \in \Downarrow\left(\left(p^{*}, \omega\right)\right)$. Because $p_{i} \sqsubseteq s \ll t$ and $n_{i}<m$ we have $\left(p_{i}, n_{i}\right) \preceq(t, m)$, so that $\Downarrow\left(\left(p^{*}, \omega\right)\right)$ is directed. It follows that $\sup \left(\Downarrow\left(\left(p^{*}, \omega\right)\right)\right)$ exists in $Q$. Obviously $\sup \left(\Downarrow\left(\left(p^{*}, \omega\right)\right)\right) \preceq\left(p^{*}, \omega\right)$.

Now consider the directed set $E^{*}$ in Step 3. For each $(p, n) \in E^{*}$ we have $p \in \Downarrow\left(p^{*}\right)$ so that $(p, n) \preceq\left(p^{*}, \omega\right)$. Apply Step 3 to see that $(p, n) \ll(p, n)$ $\preceq\left(p^{*}, \omega\right)$ so that $(p, n) \in \Downarrow\left(\left(p^{*}, \omega\right)\right)$. Hence $E^{*} \subseteq \Downarrow\left(\left(p^{*}, \omega\right)\right)$ so we must have

$$
\left(p^{*}, \omega\right)=\sup \left(E^{*}\right) \preceq \sup \left(\Downarrow\left(\left(p^{*}, \omega\right)\right)\right) \preceq\left(p^{*}, \omega\right) .
$$

We conclude that $\sup \left(\Downarrow\left(\left(p^{*}, \omega\right)\right)\right)=\left(p^{*}, \omega\right)$, as required to complete Step 5 .

STEP 6: The set of maximal elements of $(Q, \preceq)$ is given by $\max (Q)=$ $\{(y, \omega): y \in Y\}$. Consider any $y \in Y \subseteq X=\max (P)$. Then $y \in \bigcap\{O(n)$ : $n<\omega\}$ so that $(y, \omega) \in Q$. Because no element of $P$ is strictly above $y$, it follows that $(y, \omega)$ is maximal in $Q$. 
Conversely, suppose that $q$ is a maximal element of $Q$. If $q=(p, n)$ with $n<\omega$, it follows from the definition of $Q$ that $\Uparrow(p) \cap Y \neq \emptyset$. Choose $y \in Y \cap \Uparrow(p)$. Then $(y, \omega) \in Q$, and $p \ll y$ gives $(p, n) \preceq(y, \omega)$, contrary to maximality of $(p, n)$ in $Q$. Hence any maximal element $q$ of $Q$ has the form $q=(p, \omega)$. Because $p \in P$ we may choose some $x(p) \in X$ with $p \sqsubseteq x(p)$. Because $(p, \omega) \in Q_{\omega}$ we know that $p \in \bigcap\{O(n): n \geq 1\}$ and therefore $p \sqsubseteq x(p)$ gives $x(p) \in X \cap \bigcap\{O(n): n \geq 1\}=Y$. Then $(x(p), \omega) \in Q$ and $q=(p, \omega) \preceq(x(p), \omega)$ so that maximality of $q$ gives $p=x(p) \in Y$, as required to complete Step 6.

STEP 7: The continuous dcpo $(Q, \preceq)$ is algebraic. Let $q \in Q$. We must show that the set $K(q)$ of compact elements of $\Downarrow(q)$ is directed and has $\sup (K(q))=q$. If $q=(p, n)$ for $n<\omega$ then $K(q)=\Downarrow(q)$, and if $q=\left(p^{*}, \omega\right)$ then the set $E^{*}$ constructed in Step 3 is directed, and has $E^{*} \subseteq \Downarrow\left(\left(p^{*}, \omega\right)\right)$ and $\left(p^{*}, \omega\right)=\sup \left(E^{*}\right)$. Finally, each element of $E^{*}$ is compact in the light of Step 2.

STEP 8: If $Y$ is topologized as a subspace of $X$, then $Y$ is homeomorphic to the subspace $\max (Q)$ of $Q$. Define $h: Y \rightarrow \max (Q)$ by $h(y)=(y, \omega)$. Then $h$ is $1-1$ and onto.

We claim that $h$ is continuous. Suppose $h(y) \in \Uparrow(q)$ for some $q \in Q$. By Step 3, $q$ cannot have $\pi_{2}(q)=\omega$ so $q$ has the form $q=(p, n)$ with $n<\omega$. Because $(y, \omega) \in \Uparrow((p, n))$ we have $(p, n) \ll(y, \omega)$ in $Q$ so that $(p, n) \preceq(y, \omega)$ and therefore $p \ll y$ in $P$. Consequently, $\Uparrow(p) \cap Y$ is a relative neighborhood of $y$ in the relativized Scott topology from $P$. Consider any $z \in \Uparrow(p) \cap Y$. We have $p \ll z$ in $P$ so that $(p, n) \preceq(y, \omega)$ in $Q$. Because of Step 4 , we have $(p, n) \ll(p, n) \preceq(z, \omega)$ so that $h(z) \in \Uparrow((p, n))$ as required.

Finally, we claim that $h$ is an open mapping. Consider any basic neighborhood $U=\Uparrow(p) \cap Y$ in the relative Scott topology on $Y$. We must show that $h(U)$ is a relative neighborhood of $h(y)$ in $\max (Q)$. We know that $y \in Y \subseteq O(1)$ so that because $y=\sup (\Downarrow(y))$ we may choose $r \in \Downarrow(y) \cap O(1)$. Because $r \ll y$ we certainly have $\Uparrow(r) \cap Y \neq \emptyset$, so that $(r, 1) \in Q$. Consider the basic open set $\Uparrow(r, 1)$. We know that $r \ll y$ in $P$ so that $(r, 1) \preceq(y, \omega)$ in $Q$, and because $(r, 1) \ll(r, 1)$ in $Q$ we have $(r, 1) \ll(y, \omega)$ in $Q$. Thus $(y, \omega) \in \Uparrow((r, 1))$. To complete Step 8, we will show that $\Uparrow((r, 1)) \cap \max (Q) \subseteq$ $h(U)$, so let $q \in \Uparrow((r, 1)) \cap \max (Q)$. Then $q=(z, \omega)$ with $z \in Y$. From $(r, 1) \ll(z, \omega)$ in $Q$, we know that $r \ll z$ in $P$, so that $z \in \Uparrow(r) \cap Y=U$. Hence $q=(z, \omega) \in h(U)$ as required.

Our proof of Theorem 3.2 gives another proof of the following result that, K. Martin has told us, was originally obtained by Philip Sunderhauf.

Corollary 3.3. If a space $X$ can be represented as the space of maximal elements of some continuous dcpo $P$, then $X$ can also be represented as the space of maximal elements of some continuous dcpo $Q$ that is algebraic. 
Proof. Let $Y=X$. Then $Y$ is a $G_{\delta^{-}}$-subset of $X$ so that Theorem 3.2 applies to complete the proof.

Example 3.1 showed that, in general, the property of being represented as the space of maximal elements of some continuous dcpo is not hereditary to closed sets. However, in perfect spaces (= each closed set is a $G_{\delta}$-set) we have the following immediate consequence of Theorem 3.2:

COROLlary 3.4. If $X$ is a perfect space (= closed sets are $G_{\delta}$-sets) that can be represented as the space $\max (P)$ for some continuous dcpo, then each closed subspace $Y$ of $X$ can be represented as $\max (Q)$ for some continuous, algebraic dcpo $Q$.

We close this section by noting that any Čech-complete space can be represented as the set of maximal elements of a continuous, algebraic dcpo. Because locally compact Hausdorff spaces and completely metrizable spaces are Cech-complete, this generalizes a theorem of Edalat and Heckmann [5] that any completely metrizable space can be represented as the space of maximal elements of some continuous, algebraic dcpo, and also generalizes a theorem in [7] that any locally compact Hausdorff space can be represented as the space of maximal elements of a continuous dcpo. In addition, it answers question (vii) of K. Martin [8].

Theorem 3.5. Suppose $Y$ is a Čech-complete space. Then there is a continuous, algebraic dcpo $(P, \sqsubseteq)$ with $Y=\max (P)$.

Proof. Let $X=\beta Y$ be the Cech-Stone compactification of $Y$. Because $Y$ is Cech-complete, $Y$ is a dense $G_{\delta^{-}}$-subset of $X$. It is known (see [11]) that $X$, being compact Hausdorff, can be represented as $\max (P)$ where $P$ is a continuous algebraic dcpo. Apply Theorem 3.2 to conclude that $Y$ can also be represented as $\max (Q)$ for some continuous, algebraic dcpo.

It would be natural to ask whether Theorem 3.5 could be generalized further, by replacing $G_{\delta}$-subsets of compact Hausdorff spaces by Arkhangel'skilu's p-embedded subspaces of compact Hausdorff spaces. The answer is "No" because any metric space is p-embedded in its Cech-Stone compactification; now consider the metric space $\mathbb{Q}$.

The converse of Theorem 3.5 holds for metrizable spaces (see Theorem 1.1) but is false for other types of spaces, e.g., for Moore spaces. A result announced by G. M. Reed in a conference talk shows that M. E. Rudin's space in [6] is domain-representable but not Cech-complete. (In fact every Rudin-complete space (see [1]) is domain-representable.) The converse of 3.5 is also false among generalized ordered spaces, as Examples 2.2, 2.3, and 2.4 show.

Compact Hausdorff spaces can be represented as the space $\max (P)$ for some $S$ cott domain $(P, \sqsubseteq)$, i.e., a continuous dcpo $P$ with a least element 
and with the additional property that if $p_{1}, p_{2} \in P$ have $p_{1}, p_{2} \sqsubseteq p_{3}$ for some $p_{3} \in P$, then $\sup \left\{p_{1}, p_{2}\right\}$ exists in $P$ (see [11]). It is always possible to add a single minimal element to any dcpo $(Q, \preceq)$, and one might wonder whether some variation of the construction in Theorem 3.2 could show that any Cechcomplete space can be represented as $\max (R)$ for some Scott domain $R$. That cannot be done because of another example announced by G. M. Reed in several talks. He has constructed a Čech-complete Moore space that is not the space of maximal elements of any Scott domain.

EXAMPLE 3.6. There is a metrizable space $X$ that has a dense subspace $Y$ that is domain-representable, and yet $X$ is not.

Proof. Let

$$
Y=\{(k / n, 1 / n): k, n \in \mathbb{Z} \text { and } n>0\} \quad \text { and } \quad X=Y \cup\{(q, 0): q \in \mathbb{Q}\},
$$

and topologize $X$ as a subspace of the plane. (This is the space of Exercise 14, p. 253, in [2].) Then $Y$, being a set of isolated points, is completely metrizable, and is dense in $X$. But $X$ is not completely metrizable, because it has $\mathbb{Q}$ as a closed subspace. According to Theorem 1.1, $Y$ is domain-representable, but $X$ is not.

REMARK 3.7. It is well known that open mappings and perfect irreducible mappings preserve the Baire space property and that perfect mappings do not (e.g., the projection $\pi(x, y)=(x, 0)$ with the space $X$ of Example 3.6 as domain is a perfect mapping that does not preserve the Baire space property). It would be interesting to know the extent to which various types of mappings preserve the property of domain-representability. A closely related question is whether the product space $X \times K$ must be domain-representable, given that $X$ is domain-representable and $K$ is a compact Hausdorff space.

\section{References}

[1] J. Aarts and D. Lutzer, Completeness properties designed for recognizing Baire spaces, Dissertationes Math. 116 (1974).

[2] N. Bourbaki, Elements of Mathematics: General Topology, Part 2, Addison-Wesley, Reading, MA, 1966.

[3] G. Choquet, Lectures on Analysis, Vol. 1, W. A. Benjamin, New York, NY, 1969.

[4] K. Ciesielski, R. Flagg and R. Kopperman, Polish spaces, computable approximations, and bitopological spaces, Topology Appl. 119 (2002), 241-256.

[5] A. Edalat and R. Heckman, A computational model for metric spaces, Theoret. Comput. Sci. 193 (1998), 53-73.

[6] M. E. Estill, Concerning abstract spaces, Duke Math J. 17 (1950), 317-327.

[7] G. Gierz, K. H. Hoffmann, K. Keimel, J. D. Lawson, M. W. Mislove and D. S. Scott, A Compendium of Continuous Lattices, Springer, Berlin, 1980. 
[8] K. Martin, Non-classical techniques for models of computation, Topology Proc. 24 (1999), 375-405.

[9] —, Ideal models of spaces, Theoret. Comput. Sci. 305 (2003), 277-297.

[10] —, Topological games in domain theory, Topology Appl. 129 (2003), 177-186.

[11] K. Martin, M. W. Mislove, and G. M. Reed, Topology and domain theory, in: Recent Progress in General Topology II, M. Hušek and J. van Mill (eds.), Elsevier, Amsterdam, 2002, 371-394.

[12] M. W. Mislove, Topology, domain theory and theoretical computer science, Topology Appl. 89 (1998), 3-59.

[13] H. Zhang, Dualities of domains, Ph.D. thesis, Mathematics Department, Tulane, 1993.

Texas Tech University

Lubbock, TX 79409, U.S.A.

E-mail: bennett@math.ttu.edu
College of William \& Mary Williamsburg, VA 23187, U.S.A. E-mail: lutzer@math.wm.edu

Received 13 May 2005;

in revised form 2 December 2005 